\title{
Detection of Subsurface Defects in Metal Materials Using Infrared Thermography; Image Processing and Finite Element Modeling
}

\author{
Shrestha Ranjit* and Won Tae Kim* ${ }^{\dagger}$
}

\begin{abstract}
Infrared thermography is an emerging approach to non-contact, non-intrusive, and non-destructive inspection of various solid materials such as metals, composites, and semiconductors for industrial and research interests. In this study, data processing was applied to infrared thermography measurements to detect defects in metals that were widely used in industrial fields. When analyzing experimental data from infrared thermographic testing, raw images were often not appropriate. Thus, various data analysis methods were used at the pre-processing and processing levels in data processing programs for quantitative analysis of defect detection and characterization; these increased the infrared non-destructive testing capabilities since subtle defects signature became apparent. A 3D finite element simulation was performed to verify and analyze the data obtained from both the experiment and the image processing techniques.
\end{abstract}

Keywords: Non-destructive Testing, Infrared Thermography, Image Processing, Finite Element Simulation

\section{Introduction}

Currently, the use of STS materials has been gradually extended to various application fields such as nuclear pipes, automobile, railroad, and building structure. Increased usage of STS materials has led to an increased interest to the production processes including detection methods of subsurface defects.

Infrared thermography is a widely used noncontact, non-intrusive and non-destructive inspection technique that can be used for the quantitative determination of the sizes and locations of subsurface defects. Infrared thermography is gaining more acceptance as a fast non-contact and large area visualizing inspection technique primarily due to the recent advances in data acquisition and analysis systems. It utilizes an infrared camera to monitor and record the temperature variation over the viewed surface. The presence of a defect at a certain depth interferes with the heat flow causing local surface tempera- ture variations or any other changes in the thermal properties of the materials. The changes in the heat flow cause localized energy differences on the surface of the test object, which can be measured using an infrared detector. The thermal signatures are then used to evaluate various flaws, including leaks, cracks, debonding, corrosion, poor electrical wiring and contacts, and delamination [1-4]. The zones of interest (defects) appear sometimes as subtle signatures in the image obtained from the infrared camera. In this work, raw images are not often appropriate [5].

So for detection and characterization of subsurface defects, various data analysis methods such as MATLAB and Lab View program were used. The use of these methods has increased the infrared non-destructive testing (NDT) capabilities since subtle defects became more apparent. 3D finite element simulation (ANSYS program) was performed to verify and analyzed the data obtained from both the experiment and the image processing techniques.

[Received: January 21, 2014, Revised: March 29, 2014, Accepted: April 16, 2014] *Department of Mechanical Engineering, Kongju National University, Cheonan 330-717, Korea †Corresponding Author: kwt@kongju.ac.kr 


\section{Theory}

Infrared radiation (wavelength lies between $0.75 \mu \mathrm{m}$ and $1000 \mu \mathrm{m})$ emitted by a body is detected using an infrared detector and the information about temperature is obtained from the acquired data using Stefan-Boltzmann's law, which is expressed by [6],

$$
\frac{q}{A}=\varepsilon \sigma T^{4}
$$

where $\mathrm{q}$ is the rate of energy emission (W), A is the area of emitting surface $\left(\mathrm{m}^{2}\right), \mathrm{T}$ is the absolute temperature $(\mathrm{K}), \quad \sigma$ is the Stefan-Boltzmann's constant $\left(\sigma=5.676^{*} 10^{-8} \mathrm{~W} / \mathrm{m}^{2} \mathrm{~K}^{4}\right)$, and $\varepsilon$ is the emissivity of the material.

Thermography can be broadly classified into two categories, viz. passive and active. In passive thermography no external heating is required. Only the thermal radiation due to difference in temperature between the surrounding and specimen is monitored. The applications of passive thermography include monitoring of buildings and concrete structures, medical and biological investigations. On the other hand, in active thermography, an external heating is required and the evolution of surface temperature is monitored in the transient or stationary domain. Depending on the heating procedure, active thermography can be further subdivided into different categories such as pulsed, step heating, lock-in, vibrothermography and pulsed phase thermography [6-14].

Heat diffusion through a solid is a complex 3D problem that can be described by the Fourier's law of heat diffusion which is given by [7],

$$
\frac{\partial^{2} \mathrm{~T}}{\partial^{2} \mathrm{X}}-\frac{1}{\alpha} \frac{\partial \mathrm{T}}{\partial \mathrm{t}}=0
$$

where $\alpha$ is the thermal diffusivity of the material being inspected.

For a planar semi-infinite specimen heated by a sinusoidal heat source of fixed amplitude and frequency, treating the problem as onedimensional, the temperature $\mathrm{T}(\mathrm{C})$ can be expressed as a function of defect depth $z(\mathrm{~mm})$, time $\mathrm{t}(\mathrm{s})$ and angular frequency $\omega(\mathrm{Hz})$ as the following equations $[6,7]$,

$$
T_{(z, t)}=T_{0} \exp \left(-\frac{z}{\mu}\right) \cos \left(\frac{2 \pi z}{\lambda}-\omega t\right)
$$

where $T_{0}$ is initial temperature $\left({ }^{\circ} \mathrm{C}\right), \lambda$ is thermal wavelength (m), and $\mu$ is thermal diffusion length $(\mathrm{m})$ which is given by [7]

$$
\mu=\sqrt{\frac{2 \alpha}{\omega}}
$$

\section{Data Processing Methodology}

\subsection{Sample and Experimental Set-up}

A square shaped $(180 \mathrm{~mm} \times 18 \mathrm{~mm})$ specimen of the stainless materials (STS 304) was used in the experiment. Artificial defects (flat bottom holes) of different depths and diameters were created at the back side of the specimen. The schematic of the specimens with defect locations is shown in Fig. 1. The detailed geometrical descriptions of the defects in the specimens are provided in Table 1 .

Table 1 Geometrical description of defects

\begin{tabular}{c|c|c}
\hline Hole Number & Diameter $(\mathrm{mm})$ & Depth $(\mathrm{mm})$ \\
\hline A1 & 16 & 8 \\
\hline A2 & 4 & 8 \\
\hline A3 & 8 & 8 \\
\hline A4 & 12 & 8 \\
\hline B1 & 16 & 5 \\
\hline B2 & 4 & 5 \\
\hline B3 & 8 & 5 \\
\hline B4 & 12 & 5 \\
\hline C1 & 16 & 7 \\
\hline C2 & 4 & 7 \\
\hline C3 & 8 & 7 \\
\hline C4 & 12 & 7 \\
\hline D1 & 16 & 6 \\
\hline D2 & 4 & 6 \\
\hline D3 & 8 & 6 \\
\hline D4 & 12 & 6 \\
\hline & &
\end{tabular}




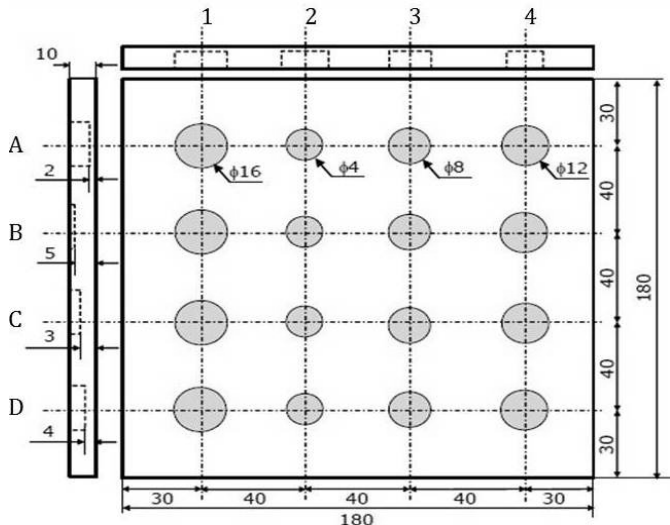

Fig. 1 Inclusion defect specimen of STS 304

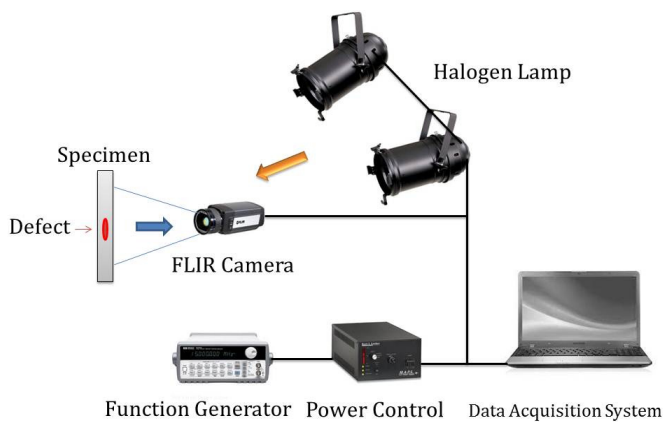

Fig. 2 Experimental set-up for active thermography

For generation of sine waves of a single frequency, a programmable function generator, (Agilent 33210A, Malaysia) was used and for detection of thermal waves infrared camera (A655SC, FLIR Systems, Sweden) was used that has a $640 \times 480$ pixels resolution. $0.68 \mathrm{mrad}$ IFOV and thermal sensitivity of $0.05{ }^{\circ} \mathrm{C}$. The sample was heated with a sinusoidally modulated heat wave from two, $1 \mathrm{~kW}$ halogen lamps (connected to the output of function generator) kept at $1000 \mathrm{~mm}$ away from the specimen. The camera was kept at a distance of $500 \mathrm{~mm}$ from the specimen in such a way that the axis of the camera coincides with the axis of the sample. Reflection method was adopted, i.e., images were acquired from the same surface that was heated periodically. Images were acquired by using FLIR R\&D software as shown in Fig. 2. The general procedure for the data acquisition is as shown in Fig. 3.

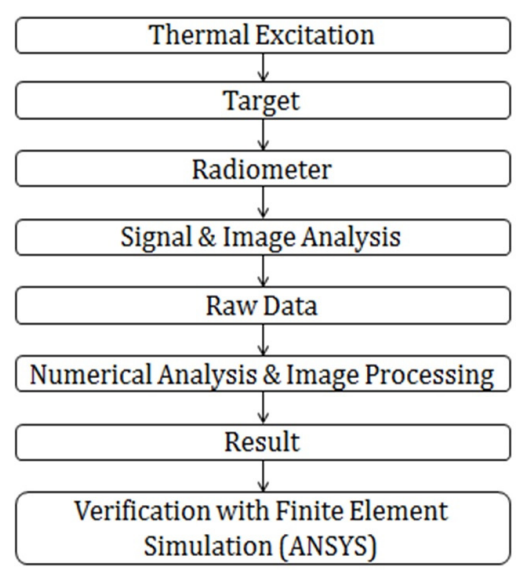

Fig. 3 Procedure for data acquisition

In thermography, temperature is interpreted from the radiation emitted from the surface under investigation. If the emissivity of the surface is very low, then the radiation emitted from this surface is very weak. Low emissivity surfaces also suffer from the problem of secondary reflection, due to the presence of surrounding bodies. The most common methodology to overcome the above mentioned emissivity problems is by applying a uniform black paint of high emissivity value, which not only increases the surface emissivity value but also reduces the secondary reflections, besides providing a uniformly emissive surface. So for the detection sensitivity, KRYLON flat black paint (emissivity 0.95) was applied to the surface of the specimen facing the camera in order to satisfy the conditions similar to the black body with an emissivity of 1 , and the emissivity to be maintained at 0.95 .

\subsection{Numerical Model:}

The physical model assumed 3D heat transfer conditions within a homogeneous STS plate. The thermal properties and geometrical param- eters are defined for the FE-model. Boundary conditions included the heat transfer by conduction on all samples boundaries. The initial 


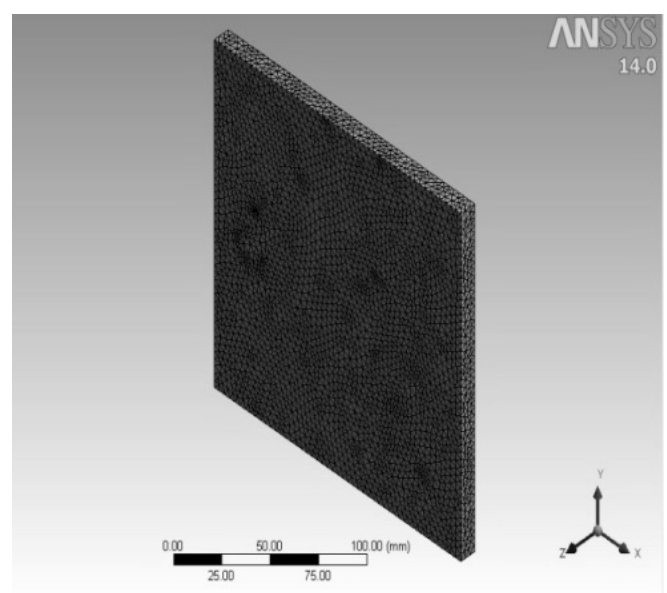

Fig. 4 Finite element model with meshing using ANSYS

Table 2 General parameters of STS 304 sample

\begin{tabular}{r|l|c|c}
\hline S.N. & \multicolumn{1}{|c|}{ Parameters } & Value & Unit \\
\hline \hline 1 & Length $(\mathrm{X})$ & 180 & $\mathrm{~mm}$ \\
\hline 2 & Length $(\mathrm{Y})$ & 180 & $\mathrm{~mm}$ \\
\hline 3. & Length $(\mathrm{Z})$ & 10 & $\mathrm{~mm}$ \\
\hline 4. & Mass $(\mathrm{m})$ & 2.5309 & $\mathrm{~kg}$ \\
\hline 5 & Volume (v) & $3.142 \mathrm{e}+005$ & $\mathrm{~mm}{ }^{3}$ \\
\hline 6 & Thermal Conductivity $(\mathrm{k})$ & $13.8 \mathrm{e}-002$ & $\mathrm{w} / \mathrm{mm}^{\circ} \mathrm{C}$ \\
\hline 7 & Specific Heat $(\mathrm{C})$ & 480 & $\mathrm{~J} / \mathrm{kg} /{ }^{\circ} \mathrm{C}$ \\
\hline 8 & Density $(\rho)$ & $8.055 \mathrm{e}-006$ & $\mathrm{~kg} / \mathrm{mm}^{3}$ \\
\hline 9 & Initial Temperature & 20 & ${ }^{\circ} \mathrm{C}$ \\
\hline 10 & Temperature Difference & 2 & ${ }^{\circ} \mathrm{C}$ \\
\hline 11 & Heat Flux $(\mathrm{Q})$ & $2.76 \mathrm{e}-003$ & $\mathrm{w} / \mathrm{mm}^{2}$ \\
\hline 12 & Nodes & 85331 & $\mathrm{No}$. \\
\hline 13 & Elements & 54080 & $\mathrm{No}$. \\
\hline
\end{tabular}

condition used in the model was taken to the room temperature as measured prior to the experiment. Assuming the temperature rise of 2 degree centigrade in the specimen, the total heat flux was calculated. Then the calculated heat flux and the initial temperature conditions were applied as the boundary condition for finding the surface temperature distribution on the specimen. The finite element model of the specimen is shown at Fig. 4. The general parameters and thermo-physical properties of the specimen are provided in Table 2.

\section{Results and Discussions}

From the experiment data, defects were only visible when the thermal diffusion length is of the order of defect depths. So a range of frequencies were used for detecting defects at various depths in the specimen. Thermography based on optical techniques provides very good defect resolution. However, results were strongly affected by surface features. For example, Fig. 5 shows the experimental image taken at frequency of $10 \mathrm{mHz}$ and Fig. 6 shows the temperature profile of path $\mathrm{X}-\mathrm{X}^{\prime}$ of Fig. 5. It was observed that visible defective holes $A_{1}, A_{4}$, $\mathrm{C}_{1}$, and $\mathrm{C}_{4}$ have the highest contrast. So it was found that the thermal contrast increases with the increased defects depth.

Image processing algorithm such as edge detection and thresholding as shown in Fig. 7, 8 and 9, were used for the raw image in MATLAB 7.10.0 (R2010a) environment. Edge detection algorithm was used for identifying and locating sharp discontinuities in an image. The dis- continuities are due to the abrupt changes in pixel intensity which characterize boundaries of objects in an image. It is also observed that Canny's edge detection algorithm performs better. Thresholding algorithm was used for image segmentation, which separates the light and dark regions in the image. These binary images provide better insights in to the defect locations and the relative contrast corresponding to different defects. From Fig. 9, it was observed that defects $A_{1}, A_{4}, C_{1}$ has the highest contrast as these were visible in the binary images even after the threshold level is set to 0.5. Such binary images may help in the development of automatic defect recognition algorithms for thermal images.

A finite element modeling scheme using ANSYS is proposed to completely simulate the active infrared thermography applied to STS plate. With the assumption of steady state 


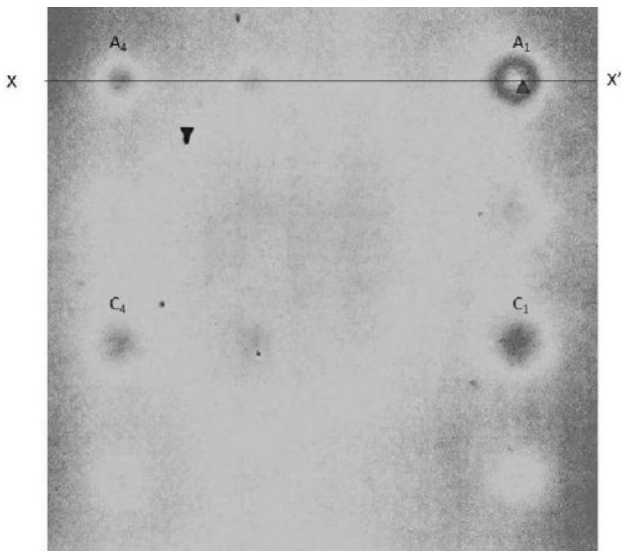

Fig. 5 Original image at frequency $10 \mathrm{mHz}$

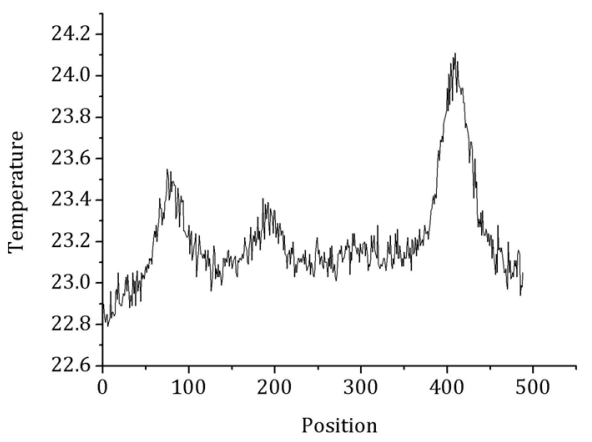

Fig. 6 Temperature profile along path $X-X^{\prime}$ of Fig. 5

condition, boundary conditions included the heat transfer by conduction on all samples boundaries. The initial condition used in the model was taken at room temperature and was measured prior to the experiment. Assuming the temperature rise of $2{ }^{\circ} \mathrm{C}$ in the specimen, the total heat flux was calculated. Then the calculated heat flux and the initial temperature conditions were applied as the boundary condition for finding the surface temperature distribution on the specimen. Fig. 10 and Fig. 11 show the surface temperature distributions of STS 304 FE-model.

As per the experiment result as shown in Fig. 5 and the finite element simulation results from Fig. 10, it is found that the defects $A_{1}$ and $\mathrm{C}_{1}$ with diameter $16 \mathrm{~mm}$ and defects depth



Fig. 7 Edge detection using Canny function in MATLAB

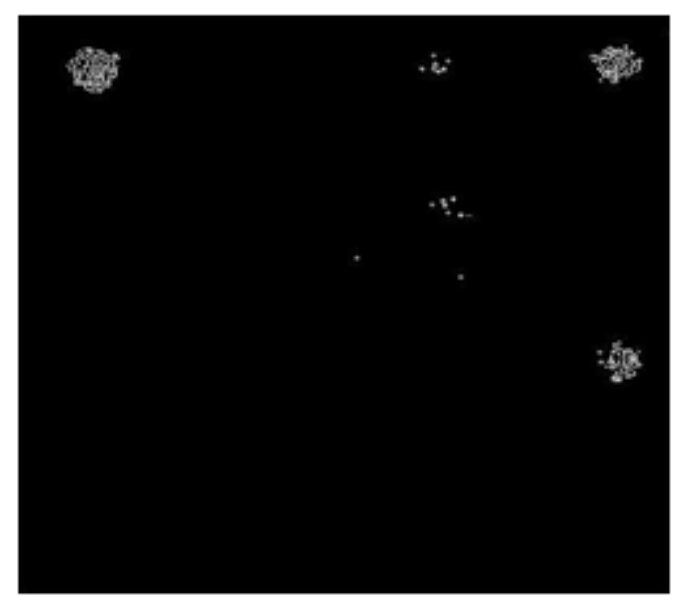

Fig. 8 Edge detection using Shobel function in MATLAB

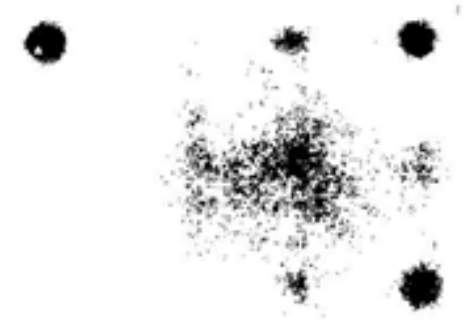

Fig. 9 Binary Image with threshold level 0.5 in MATLAB 


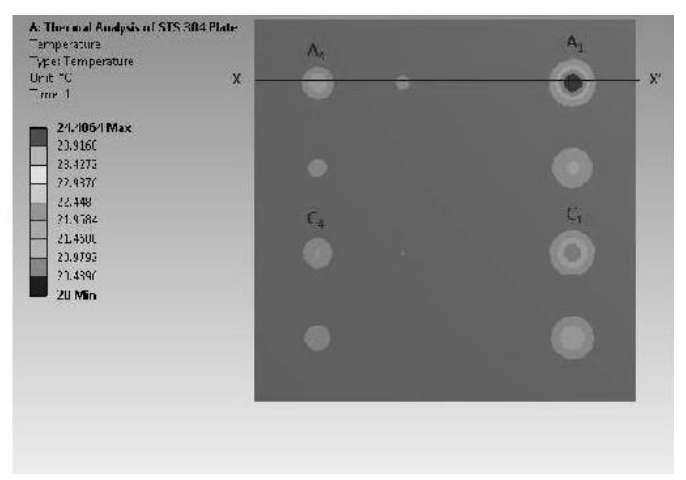

Fig. 10 FE simulation result from ANSYS

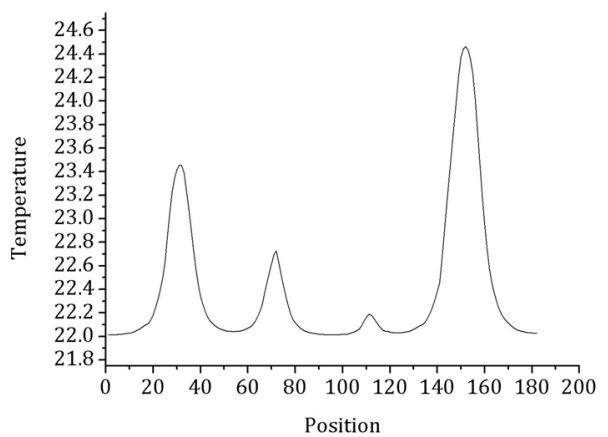

Fig. 11 Temperature profile along path $X-X^{\prime}$ of Fig. 10

$8 \mathrm{~mm}$ and $7 \mathrm{~mm}$ respectively seems more detectable while the defects with diameter $4 \mathrm{~mm}$ and $8 \mathrm{~mm}$ could not be detected.

\section{Conclusion}

Every material responds differently to thermal excitation depending on the way it has been stimulated. Thermography based on optical techniques provides very good defect resolution. However, results were strongly affected by surface features. Advanced signal and image processings were required to reduce their impact. As per the experimental result, defect detection using thermography showed that the thermal contrast increases with defect depth, while thermal contrast depended on the variation of the defect depth. It increase in defect depth and decreases with decrease in defect depth. Defects with radius to depth ratio greater than 1.0 or equal to 1 were detectable using thermography. A finite element modeling scheme using ANSYS was proposed to completely simulate the infrared thermography applied to STS plate. With the assumption of steady state condition and concerning the thermal response of defective region the numerical results were in a good agreement with experimentally achieved data from infrared thermography.

\section{Acknowledgment}

This work was supported by the Science Research Program through the National Research Foundation of Korea (NRF) funded by the Ministry of Education, Science and Technology (NRF-2011-0015969) and, by the Radiation Technology Development Program of the National Research Foundation (NRF) funded by the Ministry of Science, ICT \& Future Planning (No. 2013M2A2A9043706).

\section{References}

[1] C. Ibarra-Castanedo and X. P. V Maldague, "Infrared Thermography" Handbook of Technical Diagnostics, Springer Berlin Heidelberg, pp. 175-220 (2013)

[2] X. P. V. Maldague, "Lock-in thermography," Theory and Practice of Infrared Technology for Non-destructive Testing, pp. 355-362 (2001)

[3] X. P. V. Maldague and P. O. Moore, "Lockin thermography," Nondestructive Testing Handbook, Third Edition, Vol. 3, pp. 318-327 (2001)

[4] M. Yuan, H. Wu, Z. Tang, H. J. Kim, S. J. Song and J. Zhang, "Prediction of the effect of defect parameters on the thermal contrast evolution during flash thermography by finite element method," Journal of the Korean Society for Nondestructive Testing, Vol. 34, No. 1, pp. 10-17 (2014) 
[5] C. Ibarra-Castanedo, D. Gonzalez, M. Klein, M. Pilla, S. Vallerand and X. Maldague, "Infrared image processing and data analysis," Infrared Physics \& Technology, Vol. 46, pp. 75-83 (2004)

[6] B. B Lahiri, S. Bagavathiappan, P. R. Reshmi, J. Philip, T. Jayakumar and B. Raj, "Quantification of defects in composites and rubber materials using active thermography," Infrared Physics \& Technology, Vol. 55, pp. 191-199 (2012)

[7] H. S. Park, M. Y. Choi, J. H. Park, W. T. Kim and W. J. Choi, "Study on the qualitative defects detection in composites by optical infrared thermography," Journal of the Korean Society for Nondestructive Testing, Vol. 31, No. 2, pp. 150-156 (2011)

[8] L. Junyan, W. Yang and D. Jingmin, "Research on thermal wave processing of lock-in thermography based on analyzing image sequences for NDT," Infrared Physics \& Technology, Vol. 53, pp. 348357 (2010)

[9] M. Y. Choi, K. S. Kang, J. H. Park, W. T. Kim and K. S. Kim, "Defect sizing and location by lock-in photo-infrared thermography," Journal of the Korean Society for Nondestructive Testing, Vol. 27, No. 4, pp. 321-327 (2007)

[10] M. Y. Choi, K. S. Kang, J. H. Park, W.
T. Kim and K. S. Kim, "Quantitative determination of a subsurface defect of reference specimen by lock-in thermography," NDT\&E International, Vol. 41, pp. 119-124 (2008)

[11] G. S. Kim, G. H. Kim, J. M. Park, D. Y. Kim and B. K. Cho, "Application of infrared lock-in thermography for the quantitative evaluation of bruises on pears," Infrared Physics \& Technology, Vol. 63, pp. 133-139 (2013)

[12] D. Wu and G. Busse, "Lock-in thermography for non-destructive evaluation of materials," Rev. Gen. Therm, Vol. 37, pp. 693-703 (1998)

[13] C. Ibarra-Castanedo, M. Genest, J. M. Piau, S. Guibert, A. Bendada and X. P. V. Maldague, "Active infrared thermography techniques for the nondestructive testing of materials," Chapter XIV of the book: Ultrasonic and Advanced Methods for Nondestructive Testing and Material Characterization, Ed. Chen $\mathrm{CH}$, pp. 325-348 (2007)

[14] K. S. Kim, S. P. Yang, S. S. Kim, K. S. Kang, H. M. Kim and M. K. Kim, "Defect detection of Al specimen of using lock-in photo-infrared thermography technique," 17th World Conference on Nondestructive Testing, Shanghai, China (2008) 